\title{
Streamline topologies near a non-simple degenerate critical point close to a stationary wall using normal forms
}

\author{
By F. GÜRCAN ${ }^{1} \dagger$, A. DELİCEOĞLU ${ }^{1}$ AND P. G. BAKKER \\ ${ }^{1}$ Department of Mathematics, Erciyes University, Kayseri, Turkey 38039 \\ ${ }^{2}$ Faculty of Aerospace Engineering, Delft University of Technology, \\ Kluyverweg 22629 HS Delft, The Netherlands
}

(Received 4 March 2004 and in revised form 29 March 2005)

Streamline patterns and their bifurcations in two-dimensional Navier-Stokes flow of an incompressible fluid near a non-simple degenerate critical point close to a stationary wall are investigated from the topological point of view by considering a Taylor expansion of the velocity field. Using a five-order normal form approach we obtain a much simplified system of differential equations for the streamlines. Careful analysis of the simplified system gives possible bifurcations for non-simple degeneracies of codimension three. Three heteroclinic connections from three on-wall separation points merge at an in-flow saddle point to produce two separation bubbles with opposite rotations which occur only near a non-simple degenerate critical point. The theory is applied to the patterns and bifurcations found numerically in the studies of Stokes flow in a double-lid-driven rectangular cavity.

\section{Introduction}

The use of dynamical systems to qualitatively describe streamline patterns has a long history; for basic ideas see for example Hunt et al. (1978) and the review paper by Tobak \& Peake (1982). Early studies on separation and attachment were performed by Oswatitsch (1958) and Legendre (1948). Davey (1961) and Lighthill (1963) studied flow around obstacles and Dallmann \& Gebing (1994) considered flow attachment at flow separation lines. The topological view point was applied to the wake flow behind bluff bodies by Perry, Chong \& Lim (1987).

In the present paper, using qualitative theory we make a local analysis of the velocity field based on a Taylor series expansion of the streamfunction close to a nonsimple degenerate critical point. The coefficients in the Taylor series will be considered as bifurcation parameters. The topological structure of the fluid flow generated by the flow field may change qualitatively as parameters or boundary conditions are varied. As the flow field changes through a degenerate configuration that is structurally unstable, the streamline patterns can bifurcate and new structures arise. However, note that in the present study, for each value of the parameters, there is always a unique stable steady velocity field, but as the parameter is varied bifurcations in

$\dagger$ Author to whom correspondence should be addressed: Department of Mathematics, Erciyes University, Kayseri, Turkey 38039; gurcan@erciyes.edu.tr 
streamline patterns (i.e. for the ordinary differential equations $\dot{\boldsymbol{x}}=\boldsymbol{u}$ ) occur. It is the bifurcations of these equations considered as a dynamical system that we will explore.

Bifurcations near a critical point on a stationary plane and in a three-dimensional flow pattern were studied by Bakker (1989) and Dallmann (1983), respectively. Brøns \& Hartnack (1999) and Hartnack (1999) studied the possible flow structure in a two-dimensional incompressible flow away from boundaries and close to a fixed wall, respectively. They used normal form transformations which result in a much simplified system of differential equations for streamlines. The streamline topologies close to free and viscous surfaces in a cylinder with a rotating bottom were studied by Brøns, Voigt \& Sorensen (2001). Ma \& Wang (1999) studied topologies of twodimensional incompressible flows and applications to geophysical fluid dynamics. Ghil, Ma \& Wang (2001) showed precisely how a structural bifurcation of one-parameter families of two-dimensional incompressible flows occurs at a degenerate critical point on the boundary satisfying some natural conditions. By using a parameterization method Bakker (1989) classified flow structures at the rear of the circular cylinder in steady flow without having to consider non-generic subspaces of the parameter space. Brøns \& Hartnack (1999) and Hartnack (1999) stated that the method of Bakker is unclear.

The normal form calculations determine the codimension of the degeneracies. Using this theory Hartnack (1999) obtained a normal form of the streamline topologies for simple degenerate critical points near a fixed wall whereas for a non-simple degeneracy he stated that the normal form approach will not work and the canonical transformation was used. Here we extend Harnack's method for the simple degeneracy to the non-simple degeneracy near a fixed wall and show that for a non-simple degeneracy, the normal form approach for the order 5 works for codimension three. From this a dynamical system is obtained which is almost the same as in Bakker (1989). By using Bakker's descriptions we give a classification of flow structures that can be also observed in Stokes flow within a double-lid-driven cavity.

The double-lid-driven rectangular cavity with two moving lids and two solid walls has been considered by Weiss \& Florsheim (1965) who investigated Stokes flow in such a cavity both experimentally and theoretically for speed ratio $S=1$ (i.e. two lids moving in the same direction with equal speed). They expanded the streamfunction in terms of a set of functions which satisfy the boundary conditions on the upper and lower lids, and employed a variational principle to generate a solution to the biharmonic equation. Their solution showed that for the cavity aspect ratio $A$ (depth to width) $=0.5$ and 1 there are two eddies of equal size; for $A=2$ and 3 there are four eddies, and six eddies for $A=4$. Using a biorthogonal series expansion, Sturges (1986) found that for $S=-1$ (i.e. two lids moving in opposite directions with equal speed) and $A \geqslant 2.4$ separation bubbles (small sideeddies) appear along the sidewalls. As $A$ was increased these side eddies were found to grow in size and merge to establish a new eddy. Gürcan (2003) considered the same problem and confirmed the results for $A>1$ whereas for $A \rightarrow 0$ he used both an analytic solution for the streamfunction and methods from nonlinear dynamics for the velocity field. He showed that bifurcations occur only at the origin and give rise to separatrices with three sub-eddies (three centres and two saddles) and hence the flow structure consists of a series of nested separatrices, each contained within the central section of the previous one.

Chien, Rising \& Ottino (1986), Leong \& Ottino (1989a,b), Ottino (1989), Aref (1991), Kusch \& Ottino (1992) and Jana, Metcalfe \& Ottino (1994) studied the mixing of flows in rectangular cavities with two moving lids both experimentally and numerically. In general, they found that for $A \leqslant 1$ and $S<0$ a single eddy occupies the 
cavity whereas for $S>0$ a double eddy structure was seen. Meleshko (1996) considered a Fourier series approach to examine the double-lid-driven cavity with non-uniform velocity distributions on the lids. Gürcan et al. (2003) used an infinite series of Papkovich-Faddle eigenfunctions for the streamfunction to investigate possible flow transformations for two parameters in the range $A \in(0,3.2)$ and $S \in[-1,0)$. Here for the same cavity problem, flow transformations are investigated in the light of the theoretical results described in $\S 2$ as $A$ is increased beyond $A=4$ with $S \in\left(-1,10^{-4}\right)$.

\section{Streamline topologies near a non-simple degenerate critical point}

We consider an incompressible two-dimensional flow near a stationary wall. A streamfunction $\Psi$ exists such that the streamlines are found from

$$
\dot{x}=u=\frac{\partial \Psi}{\partial y}, \quad \dot{y}=v=-\frac{\partial \Psi}{\partial x} .
$$

To determine local information about the flow close to a given point which is taken as the origin, $\Psi$ is expanded in a power series,

$$
\Psi=\sum_{i, j=0}^{\infty} a_{i, j} x^{i} y^{j} .
$$

In this section we consider the coefficients of the series at some finite order so the analyticity of $\Psi$ is not important. We consider the $x$-axis as a stationary wall and investigate topologies under the assumption of the zero-flux and no-slip conditions, $\Psi(x, 0)=0$ and $(\partial \Psi / \partial y)(x, 0)=0$, respectively. Applying these to the streamfunction (2) gives

$$
\Psi=y^{2}\left(\sum_{i, j=0}^{\infty} a_{i, j+2} x^{i} y^{j}\right) .
$$

The above discussions are based on the existence of a streamfunction for which only incompressibility is assumed. Since we consider the Navier-Stokes equation near a stationary wall, it is necessary to determine whether the equation in steady flows can impose any restrictions on (3). This can be done by inserting expression (3) into the vorticity transport equation

$$
\nu \nabla^{4} \Psi=\frac{\partial \nabla^{2} \Psi}{\partial t}+\frac{\partial \Psi}{\partial y} \frac{\partial}{\partial x}\left(\nabla^{2} \Psi\right)-\frac{\partial \Psi}{\partial x} \frac{\partial}{\partial y}\left(\nabla^{2} \Psi\right),
$$

which can be obtained by eliminating the pressure in the Navier-Stokes equation, and collecting terms to yield a series of algebraic equations for the $a_{i, j}$. The equations of order zero and one are given by

$$
\left.\begin{array}{ll}
x^{0} y^{0}: & a_{2,2}+3 a_{0,4}=0, \\
x^{1} y^{0}: & a_{3,2}+a_{1,4}=0, \\
x^{0} y^{1}: & v\left(a_{2,3}+5 a_{0,5}\right)=\frac{1}{6} a_{1,2} a_{0,2},
\end{array}\right\}
$$

where $v$ is the kinematic viscosity. In our study only the condition of the coefficient of order zero will be used in (3). Note when (3) is inserted into the Stokes equation (in the limit $v \rightarrow \infty$ in (4)) we obtain the same equation as in that of order zero in (5). This means that all topologies described in this work can occur in steady Stokes flow. However, when higher-order coefficients are used, combinations of $a_{i, j}$ for Stokes flow are different from (5). 
The linear approximation of the equations for the streamlines is

$$
\left(\begin{array}{l}
\dot{x} \\
\dot{y}
\end{array}\right)=\left(\begin{array}{c}
2 a_{0,2} \\
0
\end{array}\right)+\left(\begin{array}{cc}
2 a_{1,2} & 3 a_{0,3} \\
0 & -a_{1,2}
\end{array}\right)\left(\begin{array}{l}
x \\
y
\end{array}\right),
$$

with the Jacobian

$$
\boldsymbol{J}=\left(\begin{array}{cc}
2 a_{1,2} & 3 a_{0,3} \\
0 & -a_{1,2}
\end{array}\right)
$$

If $2 a_{0,2}=0$ in (6), the origin is a critical point. If $\operatorname{det}(\boldsymbol{J})=0$ the critical point is degenerate and higher-order terms are needed to determine the local pictures of streamline patterns. There are two distinct subcases. The first, when eigenvalues of (7) are zero, will be referred to as a simple degeneracy, and the second, when $\boldsymbol{J}$ is the zero matrix, will be referred to as a non-simple degeneracy, see Brøns \& Hartnack (1999). If $a_{1,2}=0$ in (7), the critical point becomes a simple degenerate critical point which was investigated by Hartnack (1999). He also considered the non-simple degeneracy with codimension two by using the canonical transformation with $a_{0,2}=a_{1,2}=a_{0,3}=0$.

Here, using the normal form theory, the method of determining streamline topologies for the simple degeneracy is extended to the non-simple degeneracy with the assumptions $a_{0,2}=a_{1,2}=a_{0,3}=0$ and $a_{2,2}=0$. The last condition increases the degeneracy with codimension three and allows us more possible flow patterns near the non-simple degenerate point. From the assumptions and the equation of order zero in (5), one obtains the streamfunction

$$
\Psi=y^{2}\left(a_{1,3} x y+a_{3,2} x^{3}+a_{2,3} x^{2} y+a_{1,4} x y^{2}+a_{0,5} y^{3}\right)+O\left((x, y)^{6}\right) .
$$

For a significant simplification of higher-order terms in the streamfunction and easy determination of the codimension, we use the normal form theory in the next section.

\subsection{The normal form transformation}

The successive transformations from the Hamiltonian systems to a much simplified system of differential equations for the streamlines (a normal form) encapsulating all the evolution of the original system can be performed by using canonical transformations with generating functions, see Brøns \& Hartnack (1999) and Hartnack (1999). It will be shown how to simplify terms of fifth order in (8). The new coordinates $(\xi, \eta)$ are found from a canonical transformation defined by the generating function

$$
S=y \xi+\sum_{i+j=3} s_{i, j} y^{i} \xi^{j}
$$

such that

$$
x=\frac{\partial S}{\partial y}, \quad \eta=\frac{\partial S}{\partial \xi} .
$$

Solving (10) for $x, y$ and including terms up to the second order yields

$$
\begin{aligned}
& x=\xi+s_{1,2} \xi^{2}+2 s_{2,1} \xi \eta+3 s_{3,0} \eta^{2}, \\
& y=\eta-3 s_{0,3} \xi^{2}-2 s_{1,2} \xi \eta-s_{2,1} \eta^{2} .
\end{aligned}
$$

We require that the boundaries are preserved under the transformation, i.e. $y=0$ is mapped to $\eta=0$. This is achieved by taking $s_{0,3}=0$ in (12). Inserting this transformation in (8) and truncating after the fifth order, we obtain

$$
\begin{aligned}
\Psi= & \eta^{2}\left[a_{1,3} \xi \eta+a_{3,2} \xi^{3}+\left(a_{2,3}-5 a_{1,3} s_{1,2}\right) \xi^{2} \eta\right. \\
& \left.+\left(-a_{1,3} s_{2,1}+a_{1,4}\right) \xi \eta^{2}+\left(3 a_{1,3} s_{3,0}+a_{0,5}\right) \eta^{3}\right]+O\left((\xi, \eta)^{6}\right) .
\end{aligned}
$$




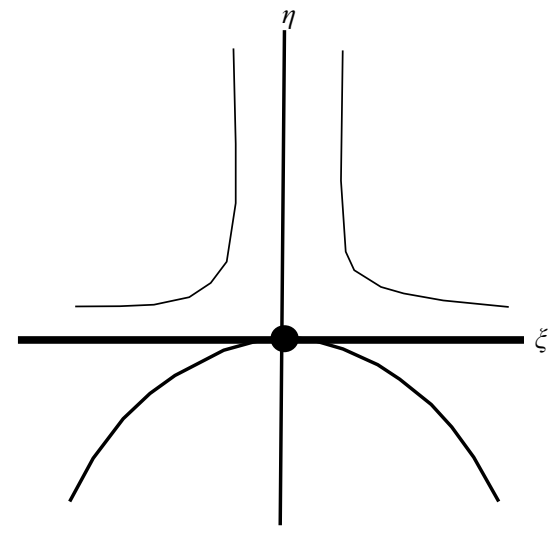

FIGURE 1. Streamline pattern near a non-simple degenerate critical point for (16).

We are free to choose the $s_{i, j}$. With the choices

$$
s_{3,0}=\frac{-a_{0,5}}{3 a_{1,3}}, \quad s_{2,1}=\frac{a_{1,4}}{a_{1,3}}, \quad s_{1,2}=\frac{a_{2,3}}{5 a_{1,3}},
$$

we eliminate a number of terms and get

$$
\Psi=\eta^{2}\left(a_{1,3} \xi \eta+a_{3,2} \xi^{3}\right)+O\left((\xi, \eta)^{6}\right) .
$$

Under the condition $a_{3,2} \neq 0$ further reduction is possible by dividing the streamfunction by $a_{3,2}$ and then scaling $\eta$ by the substitution $\eta \rightarrow\left(a_{3,2} / a_{1,3}\right) \eta$. Finally, dropping the $O$-terms in (15) gives the normal form of order 5 for $\Psi$ for an on-wall non-simple degenerate critical point and one obtains the following.

THEOREM 3.1. Let $a_{0,2}, a_{1,2}, a_{0,3}$ and $a_{2,2}$ be taken as zero. Assuming the condition $a_{1,3} \neq 0$ and $a_{3,2} \neq 0$, then the normal form for $\Psi$ is

$$
\Psi=\eta^{2}\left(\xi \eta+\xi^{3}\right) .
$$

From the normal form the local flow topology in the neighbourhood of the non-simple degenerate critical point is easily obtainable. Possible separatrices of the critical point are given by $\Psi=0$ which yields

$$
\eta+\xi^{2}=0, \quad \eta=0 \quad \text { and } \quad \xi=0 .
$$

In the physical plane $(\eta \geqslant 0)$ considered here, there is a non-simple degenerate critical point showing a saddle with two hyperbolic sectors, see figure 1 . For $\eta<0$, see Bakker (1989).

\subsection{Unfolding of a non-simple degenerate critical point}

The previous section only involved flow patterns which are structurally unstable when the parameters take certain degenerate values. Rather, we aim to find all possible patterns that may occur when the degenerate streamfunction is perturbed. To study the bifurcations close to the non-simple degenerate critical points, we introduce the small parameters,

$$
\epsilon_{1}=a_{0,2}, \quad \epsilon_{2}=a_{1,2}, \quad \epsilon_{3}=a_{0,3}, \quad \epsilon_{4}=a_{2,2},
$$

and apply normal form transformations to simplify the system, but now include the small parameters in the transformations. We briefly show how to simplify fifth-order 
terms in the streamfunction. A generating function is chosen,

$$
S=y \xi+\sum_{i+j+k+l+m+n=3} s_{i j k l m n} y^{i} \xi^{j} \epsilon_{1}^{k} \epsilon_{2}^{l} \epsilon_{3}^{m} \epsilon_{4}^{n},
$$

defining a canonical transformation through the relations (10). Solving (10) for $x, y$ and including terms up to second order yields

$$
\begin{aligned}
x= & s_{120000} \xi^{2}+2 s_{210000} \xi \eta+s_{111000} \xi \epsilon_{1}+s_{110100} \xi \epsilon_{2}+s_{110010} \xi \epsilon_{3}+s_{110001} \xi \epsilon_{4} \\
& +3 s_{300000} \eta^{2}+2 s_{201000} \eta \epsilon_{1}+2 s_{200100} \eta \epsilon_{2}+2 s_{200010} \eta \epsilon_{3}+2 s_{200001} \eta \epsilon_{4} \\
& +s_{102000} \epsilon_{1}^{2}+s_{101100} \epsilon_{1} \epsilon_{2}+s_{101010} \epsilon_{1} \epsilon_{3}+s_{101001} \epsilon_{1} \epsilon_{4}+s_{100200} \epsilon_{2}{ }^{2} \\
& +s_{100110} \epsilon_{2} \epsilon_{3}+s_{100101} \epsilon_{2} \epsilon_{4}+s_{100020} \epsilon_{3}^{2}+s_{100011} \epsilon_{3} \epsilon_{4}+s_{100002} \epsilon_{4}{ }^{2}+\xi,
\end{aligned}
$$

$y=-3 s_{030000} \xi^{2}-2 s_{120000} \xi \eta-2 s_{021000} \xi \epsilon_{1}-2 s_{020100} \xi \epsilon_{2}-2 s_{020010} \xi \epsilon_{3}-2 s_{020001} \xi \epsilon_{4}$

$-s_{210000} \eta^{2}-s_{111000} \eta \epsilon_{1}-s_{110100} \eta \epsilon_{2}-s_{110010} \eta \epsilon_{3}-s_{110001} \eta \epsilon_{4}-s_{012000} \epsilon_{1}{ }^{2}$

$-s_{011100} \epsilon_{1} \epsilon_{2}-s_{011010} \epsilon_{1} \epsilon_{3}-s_{011001} \epsilon_{1} \epsilon_{4}-s_{010200} \epsilon_{2}^{2}-s_{010110} \epsilon_{2} \epsilon_{3}$

$-s_{010101} \epsilon_{2} \epsilon_{4}-s_{010020} \epsilon_{3}^{2}-s_{010011} \epsilon_{3} \epsilon_{4}-s_{010002} \epsilon_{4}^{2}+\eta$.

As earlier, the original boundary $y=0$ is to be mapped onto $\eta=0$. This is achieved by choosing

$$
\left.\begin{array}{l}
s_{010002}=s_{010011}=s_{010020}=s_{010101}=s_{010110}=0, \\
s_{010200}=s_{011001}=s_{011010}=s_{011100}=s_{012000}=0, \\
s_{020001}=s_{020010}=s_{020100}=s_{021000}=s_{030000}=0 .
\end{array}\right\}
$$

Inserting the transformation equations (20) and (21) with (22) into the streamfunction (3), one obtains

$$
\begin{aligned}
\Psi= & \eta^{2}\left[a_{3,2} \xi^{3}+\left(a_{2,3}-5 a_{1,3} s_{120000}\right) \xi^{2} \eta+\left(-a_{1,3} s_{210000}+a_{1,4}\right) \xi \eta^{2}\right. \\
& +\left(a_{0,5}+3 a_{1,3} s_{300000}\right) \eta^{3}+\left(4 \epsilon_{1} s_{120000}{ }^{2}-3 \epsilon_{2} s_{120000}+\epsilon_{4}\right) \xi^{2} \\
& +\left(a_{1,3}-6 \epsilon_{3} s_{120000}+4 \epsilon_{1} s_{210000} s_{120000}\right) \xi \eta+\left(3 s_{300000}+2 a_{1,3} s_{200100}\right) \epsilon_{2} \eta^{2} \\
& +\left(-3 s_{210000}+2 a_{1,3} s_{200010}\right) \epsilon_{3} \eta^{2}+\left(-\frac{1}{3}+2 a_{1,3} s_{200001}\right) \epsilon_{4} \eta^{2}+\left(s_{210000}{ }^{2}\right. \\
& \left.+2 a_{1,3} s_{201000}\right) \epsilon_{1} \eta^{2}+\left(-4 \epsilon_{1} s_{120000}+\epsilon_{2}\right) \xi+\left(2 s_{201000} \epsilon_{1} \epsilon_{2}+2 s_{200010} \epsilon_{2} \epsilon_{3}\right. \\
& \left.\left.+\epsilon_{3}+2 s_{200100} \epsilon_{2}{ }^{2}+2 s_{200001} \epsilon_{2} \epsilon_{4}-2 \epsilon_{1} s_{210000}\right) \eta+\epsilon_{1}\right]+O\left((\xi, \eta)^{6} .\right.
\end{aligned}
$$

By choosing

$$
\begin{aligned}
& s_{200001}=\frac{1}{6} \frac{1}{a_{1,3}}, \quad s_{210000}=\frac{a_{1,4}}{a_{1,3}}, \quad s_{120000}=\frac{1}{5} \frac{a_{2,3}}{a_{1,3}}, \quad s_{300000}=-\frac{1}{3} \frac{a_{0,5}}{a_{1,3}}, \\
& s_{201000}=-\frac{1}{2} \frac{a_{1,4} a_{1,3}{ }^{3}}{a_{1,3}}, \quad s_{200100}=\frac{1}{2} \frac{a_{0,5}}{a_{1,3}{ }^{2}}, \quad s_{200010}=\frac{3}{2} \frac{a_{1,4}}{a_{1,3}{ }^{2}} .
\end{aligned}
$$

and setting the remaining coefficients in the generating function $S$ equal to zero, the result is

$$
\begin{aligned}
\Psi= & \eta^{2}\left[a_{3,2} \xi^{3}+\left(\frac{4}{25} \frac{\epsilon_{1} a_{2,3}{ }^{2}}{a_{1,3}{ }^{2}}-\frac{3}{5} \frac{\epsilon_{2} a_{2,3}}{a_{1,3}}+\epsilon_{4}\right) \xi^{2}+\left(a_{1,3}-\frac{6}{5} \frac{\epsilon_{3} a_{2,3}}{a_{1,3}}+\left(\frac{4}{5} \frac{\epsilon_{1} a_{1,4} a_{2,3}}{a_{1,3} 2^{2}}\right) \xi \eta\right.\right. \\
& +\left(-\frac{4}{5} \frac{\epsilon_{1} a_{2,3}}{a_{1,3}}+\epsilon_{2}\right) \xi+\left(-\frac{a_{1,4} \epsilon_{1} \epsilon_{2}}{a_{1,3}{ }^{3}}+3 \frac{a_{1,4} \epsilon_{2} \epsilon_{3}}{a_{1,3}{ }^{2}}+\epsilon_{3}+\frac{a_{0,5} \epsilon_{2}{ }^{2}}{a_{1,3}{ }^{2}} \frac{1}{3} \frac{\epsilon_{2} \epsilon_{4}}{a_{1,3}}-2 \frac{\epsilon_{1} a_{1,4}}{a_{1,3}}\right) \eta \\
& \left.+\epsilon_{1}\right]+O\left((\xi, \eta)^{6} .\right.
\end{aligned}
$$


Translation of the origin will remove the terms of the next highest degree in a polynomial by replacing $\xi$ by $\xi+\xi_{0}$, where

$$
\xi_{0}=-\frac{1}{75} \frac{4 \epsilon_{1} a_{2,3}{ }^{2}-15 \epsilon_{2} a_{2,3} a_{1,3}+25 \epsilon_{4} a_{1,3}{ }^{2}}{a_{1,3}{ }^{2} a_{3,2}} ;
$$

then we obtain

$$
\Psi=\eta^{2}\left(\overline{\overline{\mu_{1}}}+\overline{\overline{\mu_{2}}} \xi+\overline{\overline{\mu_{3}}} \eta+\overline{\overline{\mu_{4}}} \xi \eta+a_{3,2} \xi^{3}\right)+O\left((\xi, \eta)^{6},\right.
$$

where $\overline{\overline{\mu_{1}}}, \overline{\overline{\mu_{2}}}, \overline{\overline{\mu_{3}}}, \overline{\overline{\mu_{4}}}$ are new small parameters depending on $\epsilon_{1}, \epsilon_{2}, \epsilon_{3}$. A final simplification can be obtained by first dividing $\Psi$ by $a_{3,2}$ corresponding to scaling the time and then scaleing $\eta$ by the substitution $\eta \rightarrow\left(a_{3,2} / \overline{\overline{\mu_{4}}}\right) \eta$ to obtain

$$
\Psi=\eta^{2}\left(\overline{\mu_{1}}+\overline{\mu_{2}} \xi+\overline{\mu_{3}} \eta+\xi \eta+\xi^{3}\right)+O\left((\xi, \eta)^{6} .\right.
$$

Proceeding as above, in the general case (returning the coordinates back to $x, y$ ) one obtains the following.

THEOREM 3.2. Let $a_{0,2}, a_{1,2}, a_{0,3}$ and $a_{2,2}$ be small parameters. Assuming the nondegeneracy conditions $a_{1,3} \neq 0, a_{3,2} \neq 0$ are satisfied, then the normal form of order 5 for the streamfunction (3) is

$$
\Psi=y^{2}\left(\mu_{1}+\mu_{2} x+\mu_{3} y+x y+x^{3}\right),
$$

where $\mu_{1}, \mu_{2}$ and $\mu_{3}$ are small transformed parameters.

Using the normal form (27) having codimension three the corresponding dynamical system (time scaled with $y$ ),

$$
\left.\begin{array}{l}
\dot{x}=2 \mu_{1}+2 \mu_{2} x+3 \mu_{3} y+3 x y+2 x^{3}, \\
\dot{y}=-y\left(\mu_{2}+y+3 x^{2}\right),
\end{array}\right\}
$$

with the Jacobian

$$
\boldsymbol{J}=\left(\begin{array}{cc}
2 \mu_{2}+3 y+6 x^{2} & 3 \mu_{3}+3 x \\
-6 x y & -\mu_{2}-2 y-3 x^{2}
\end{array}\right),
$$

can be analysed by using the description by Bakker (1989). When $\operatorname{det}(\boldsymbol{J})=0$ on-wall bifurcation occurs for

$$
\left.\begin{array}{l}
\mu_{1}+\mu_{2} x+x^{3}=0 \\
\mu_{2}+3 x^{2}=0
\end{array}\right\}
$$

By eliminating $x$ one finds

$$
\left(\frac{\mu_{2}}{3}\right)^{3}=-\left(\frac{\mu_{1}}{2}\right)^{2}
$$

which forms a bifurcation set labelled $A_{1}$. For this curve, corresponding to a thirdorder saddle point, there are two possible unstable structures which are illustrated in figure 2, see 2 (above $B$ on $A_{1}$ ) and 10 (below $B$ ). This figure shows bifurcation sets dividing the parameter plane into several sub-domains where topologically different flow patterns occur. This is almost the same as those of Bakker and hence we omit the details except the possibility of global bifurcations such as saddle to saddle connections of off- and on-wall critical points. This requires $\Psi$ to attain the same value at the on- and off-wall critical points. Evaluating $\Psi$ at the wall and at an in-flow 


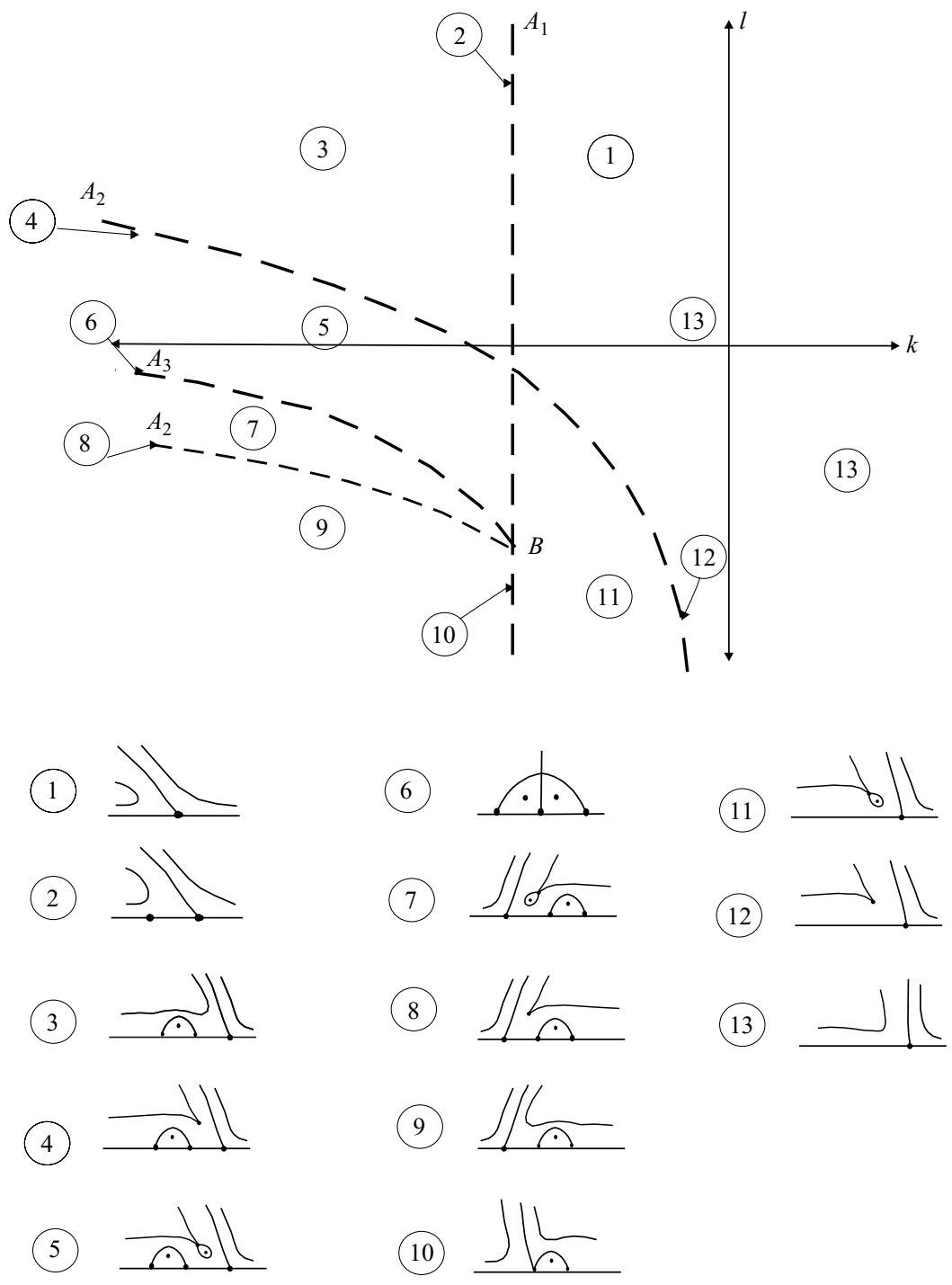

FIGURE 2. Bifurcation diagram for the fifth-order normal form and corresponding flow patterns. Parameters, $\mu_{2}=k\left(\frac{1}{2} \mu_{1}\right)^{2 / 3}, \mu_{3}=l\left(\frac{1}{2} \mu_{1}\right)^{1 / 3}$ for $\mu_{1} \neq 0$, are considered as in Bakker (1989).

critical point, $\left(-\mu_{3},-\mu_{2}+\mu_{3}^{2}\right)$, satisfying (28) gives zero and an algebraic condition

$$
\mu_{1}-\mu_{2} \mu_{3}-\mu_{3}^{3}=0 .
$$

This means that (31) has a global bifurcation curve labelled $A_{3}$, see pattern 6 in figure 2 where a separation bubble and a separating streamline interact with an in-flow saddle point creating two separation bubbles around the flow centres with opposite rotations. This pattern cannot be observed by using the unfolding of simple degeneracies which gives rise to the interaction of separation bubbles with the same rotation observed by Hartnack (1999).

In the next section, we apply the theory to describe the possible qualitative structures of the bifurcation sets in the double-lid-driven rectangular cavity problem. 


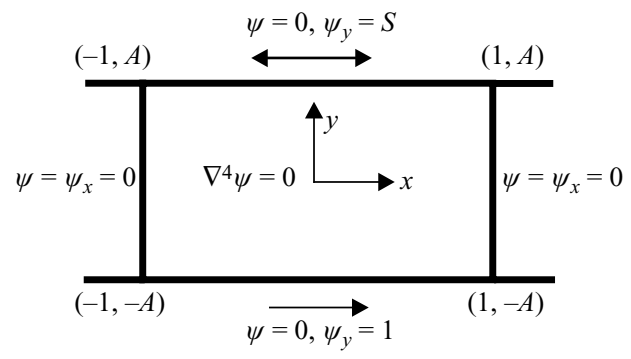

FIGURE 3. The dimensionless boundary value problem for the double-lid-driven cavity.

\section{Application}

The two-dimensional Stokes flow of an incompressible fluid in a double-lid-driven rectangular cavity with two solid walls lying along $X= \pm L$ and two moving lids lying along $Y= \pm H$ is governed by the biharmonic equation for the streamfunction $\psi$

$$
\nabla^{4} \psi=0
$$

Lengths are non-dimensionalized with respect to $L$ such that the flow domain is given by $|x| \leqslant \pm 1,|y| \leqslant \pm A$ where $A=L / H$ is the cavity aspect ratio and $S=U_{1} / U_{2}$ is the speed ratio where $U_{1}$ and $U_{2}$ are the top and bottom lid velocities, respectively (see figure 3). The streamfunction is set equal to zero on the boundaries of the closed domain, i.e.

$$
\psi( \pm 1, y)=0 \quad \text { and } \quad \psi(x, \pm A)=0
$$

and the no-slip conditions for the upper and lower lids and sidewalls are

$$
\begin{gathered}
\frac{\partial \psi}{\partial y}(x, A)=S, \frac{\partial \psi}{\partial y}(x,-A)=1, \\
\frac{\partial \psi}{\partial x}(1, y)=\frac{\partial \psi}{\partial x}(-1, y)=0 .
\end{gathered}
$$

Following Gürcan (1997) and Joseph \& Sturges (1978) the streamfunction for any value of $S$ can be written as

$$
\psi=\sum_{n=-\infty}^{\infty}\left\{A_{n} \mathrm{e}^{s_{n}(y-A)}+B_{n} \mathrm{e}^{-s_{n}(y+A)}\right\} \frac{\phi_{1}^{n}\left(x, s_{n}\right)}{s_{n}^{2}},
$$

where the complex coefficients $A_{n}$ and $B_{n}$ are determined from the boundary conditions using a truncation technique employing Smith's (1952) biorthogonality relation. The $s_{n}$ are complex eigenvalues and the functions $\phi_{1}^{n}$ are even PapkovichFaddle eigenfunctions, satisfying the no-slip boundary conditions on the sidewalls, see Gürcan (1997) for more details. When the coefficients have been determined, the streamfunction at any interior point in the liquid is obtained by simply summing a finite number of terms in the series (36) while ensuring that the magnitude of the truncation error is acceptably small, see Gürcan et al. (2003). Figures in the present paper were produced using a truncation number of $N=200$.

\subsection{Flow patterns}

We consider $(S, A)$ space in the range $-1<S<-10^{-4}$ and $4<A<5$ to obtain a number of key stages in the flow structures and to construct a bifurcation diagram exhibiting flow bifurcations at degenerate critical points. It is necessary to describe 
(a)

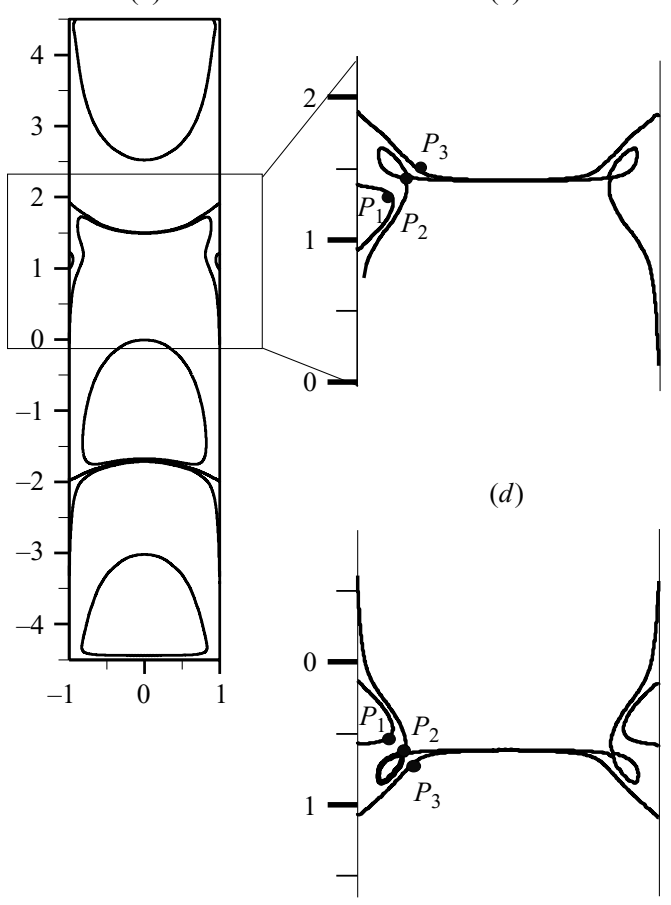

(b)

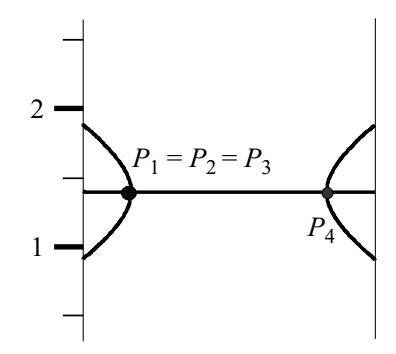

(e)

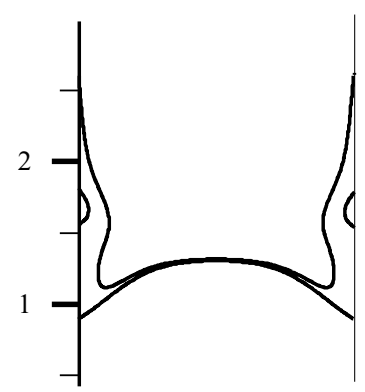

FIgURE 4. Streamline patterns for the double-lid-driven cavity with $S$ increasing and $A=4.5$. (a) $S=-0.0035,(b) S=-0.0029$, (c) $S_{c}=-0.0028$, (d) $S=-0.00268$ and $(e) S=-0.0023$.

exactly what is meant by bifurcation, since for each combination of $S$ and $A$ in the cavity problem there is always a unique flow field. At stagnation points the fluid velocity, $\boldsymbol{u}$, is zero and so such points are solutions of $\boldsymbol{u}(\boldsymbol{x}, S, A)=\dot{\boldsymbol{x}}(\boldsymbol{x}, S, A)=0$. Hence as $A$ and $S$ are changed, the number (or type) of stagnation points changes at critical values of these parameters, and in this section the term bifurcation refers to such a change in the stagnation point configuration.

We first consider $A=4.5$ for increasing $S$ from -1 to $-10^{-4}$ and obtain a sequence of flow patterns as illustrated in figure 4. In particular, figure 4(a) shows three large eddies with two small separation bubbles (side eddies) along the sidewalls of the central section. As $S$ is increased it is the flow within this central section, between the two separating streamlines, which undergoes structural change. The side eddies grow and approach the separating streamline near the upper eddy; and at $S_{1}=-0.00306$ saddle node bifurcations arise between this separating streamline and side eddies. Figure 4(b) shows the flow pattern when $S=-0.0029$. As $S$ continues to increase $P_{1}$ and $P_{3}$ in figure $4(b)$ approach the saddle point $P_{2}$ and at the critical speed ratio $S_{c}=-0.0028, P_{1}$ and $P_{3}$ merge with $P_{2}$ to produce four heteroclinic connections from each saddle point, figure $4(c)$. When $S$ is increased beyond $S_{c}$ the heteroclinic connections separate from the saddle points $P_{2}$ and $P_{4}$, see figure $4(d)$. Between each side eddy and the separating streamline there is a sub-eddy with a saddle point close to the sidewalls. As $S$ is increased the centres and saddle points approach each other, coalesce and disappear at $S_{2}=-0.00256$. Figure 4(e) shows the flow pattern for $S=-0.0023$. At $S_{3}=-0.00178$ the side eddies disappear and three eddies occupy the cavity.

The flow transformations shown in figure 4 can be tracked as $A$ and $S$ are varied and this is illustrated in an $(S, A)$ control space diagram (see figure 5) with bifurcation 


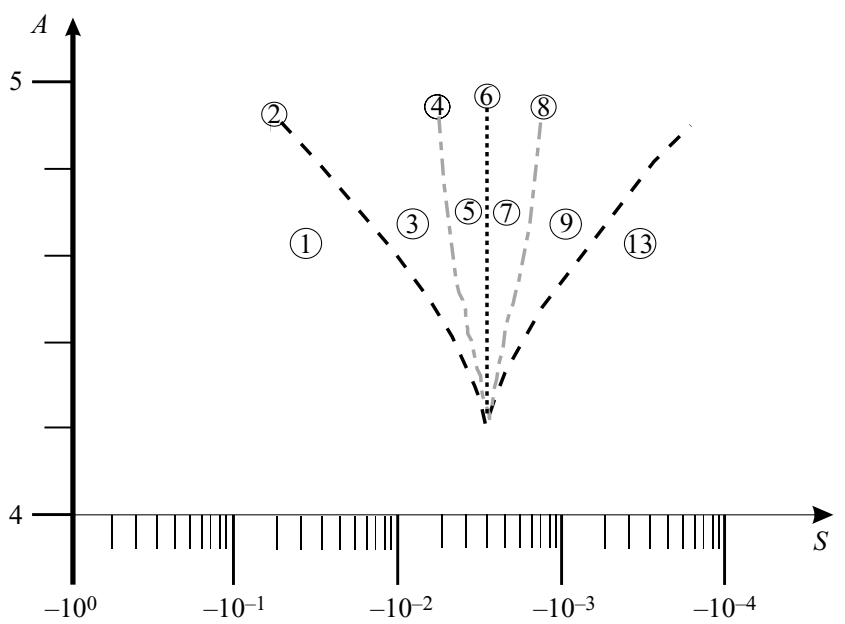

FIGURE 5. $(S, A)$ control space diagram with the bifurcations curves.

curves. Figure 5 shows a quasi-flow-symmetry about the bifurcation curve 6 with the value of $S_{c}=-0.0028$. For $S<S_{c}$ flow development in the central section follows a similar pattern to that in the upper section of the cavity for $S>S_{c}$. By variation of the speed ratio for a fixed $A$ in figure 5 one encounters most of the flow patterns and their bifurcations shown in figure 2. The flow patterns $1-9$ and 13 in figure 2 correspond exactly to the patterns in figure 5 which are labelled with the same number as those of in figure 2. However, patterns 10, 11 and 12 in figure 2 cannot appear in the cavity for the considered values of $(S, A)$ parameters, i.e. the flow configuration jumps from type 9 to type 13 in figure 5. This may be interpreted by using only two bifurcation parameters in the cavity where bifurcations with codimension up to two occur generically. Whereas in the theory three bifurcation parameters $\left(\mu_{1}, \mu_{2}, \mu_{3}\right)$ are considered to determine flow bifurcations for codimension three. If two bifurcation parameters $\mu_{2}, \mu_{3}$ are considered (take $\mu_{1}=0$ ) then the jump can also be observed. From the bifurcation equations, (30) and (31), one may simply obtain a bifurcation diagram in the $\left(\mu_{2}, \mu_{3}\right)$ parameter plane having only the flow patterns 1-9 and 13 .

\section{Conclusion}

Normal form theory provides a context for determining the codimension of degenerate patterns and their bifurcations by simplifying the dynamical system significantly. There are two types of degenerate critical points (simple and nonsimple degeneracies) which depend on the Jacobian matrix of the velocity field. We consider a non-simple degenerate critical point and develop the normal form theory for this point close to the stationary wall. A bifurcation diagram is constructed for the fifth-order normal form of the streamfunction by using the simplified dynamical systems. By considering the unfolding of fifth-order non-simple degeneracies we obtain a global bifurcation which gives rise to two separation bubbles with opposite rotations.

A topological classification provides guidelines for identification of structures in any flow. We have shown how this could be done for Stokes flow in a double-lid-driven rectangular cavity. Using an analytical solution for the streamfunction we obtain streamline patterns in the rectangular cavity. It is the flow within the central section 
of the cavity which undergoes structural change. These are similar to those obtained from the theory. We also find some structurally unstable flow patterns from the theory using three parameters whereas these patterns may not be seen in the cavity problem because two bifurcation parameters are used.

The authors are grateful to Professor M. D. Savage for his help and a reviewer for helpful suggestions.

\section{REFERENCES}

Andronov, A. A., Leontovich, E. A., Gordon, I. I. \& Maier, A. G. 1973 Qualitative Theory of Second-order Dynamical Systems. Wiley.

Aref, H. 1991 Chaotic advection of fluid particles. Phil. Trans. R. Soc. Lond. A 333, 273-292.

BAKKeR, P. G. 1989 Bifurcation in flow patterns. Thesis, Technical University of Delft, Netherlands.

Brons, M. \& Hartnack, J. N. 1999 Streamline topologies near simple degenerate critical points in two-dimensional flow away from boundaries. Phys. Fluids 11, 314-324.

Brons, M., Voigt, L. K. \& Sorensen, J. N. 2001 Topology of vortex breakdown bubbles in a cylinder with a rotating bottom and a free surface. J. Fluid Mech. 428, 133-148.

Chien, W. L., Rising, H. \& Ottino, J. M. 1986 Laminer mixing and chaotic mixing in several cavity flows. J. Fluid Mech. 170, 355-377.

Dallmann, U. 1983 Topological structures of three-dimensional flow separations. IB 221-82 Rep. A 07. DFVLR.

Dallmann, U. \& Gebing, H. 1994 Flow attachment at flow separation lines. Acta Mech. 4, 47.

DAVEY, A. 1961 Boundary-layer flow at a saddle point of attachment. J. Fluid Mech. 10, 593.

Ghil, M., MA, T. \& WANG, S. 2001 Structural bifurcation of 2-D incompressible flows. Indiana Univ. Math. J. 50, 159-180.

GÜRCAN, F. 1997 Flow bifurcations in rectangular, lid-driven, cavity flows. PhD Thesis, University of Leeds.

GürCan, F. 2003 Streamline topologies in stokes flow within lid-driven cavities. Theoret. Comput. Fluid Dyn. 17, 19-30.

Gürcan, F., Gaskell, P. H., Savage, M. D. \& Wilson, M. C. T. 2003 Eddy genesis and transformation of stokes flow in a double-lid driven cavity. Proc. Inst. Mech. Engng C J. Mech. Engng Sci. 217, 353-364.

Hartnack, J. N. 1999 Streamlines topologies near a fixed wall using normal forms. Acta Mech. 136, $55-75$.

Hunt, J. C. R., Abell, C. J., Peterka, J. A. \& Woo, H. 1978 Kinematical studies of the flow around free surface-mounted obstacles; applying topology to flow visualization. J. Fluid Mech. 86, 179.

Jana, C. M., Metcalfe, G. \& Ottino, J. M. 1994 Experimental and computational studies of mixing complex stokes flows: the vortex mixing flow and multicellular cavity flows. J. Fluid Mech. 269, 199-246.

JosePh, D. D. \& Sturges, L. 1978 The convergence of biorthogonal series for biharmonic and stokes flow edge problems: Part II. SIAM. J. Appl. Maths 34, 7-27.

Kusch, H. A. \& Ottino, J. M. 1992 Experiments on mixing in continuous flows. J. Fluids Mech. 236, 319-348.

Legendre, R. 1956 Sparation de I'coulement laminaire tridimensionel. Rech. Aerosp. 54, 3.

Leong, C. W. \& OtTino, J. M. $1989 a$ Experiments on mixing due to chaotic advetion in a cavity. J. Fluid Mech. 209, 463-499.

Leong, C. W. \& Ottino, J. M. 1989 b Gallery of fluid motion. Phys. Fluids A 1, 1441.

Lighthill, M. J. 1963 Attachment and separation in three-dimensional flow. In Laminar Boundary Layers (ed. L. Rosenhead), p. 72. Clarendon.

MA, T. \& WANG, S. 1999 Topology of 2D incompressible flows and applications to geophysical fluid dynamics. RACSAM, Rev. R. Acad. Cien. A Mat. 96(3), 447-459.

Meleshro, V. V. 1996 Steady stokes flow in a rectangular cavity. Proc. R. Soc. Lond. A 452, 1999-2022. 
OswatitsCH, K. 1958 Die ablosungsbedingung von grenzschichten. In IUTAM Symposium on Boundary Layer Research, pp. 357-364. Springer.

Ottino, J. M. 1989 The mixing of fluids. Sci. Am. Jan., 40-50.

Perry, A. E., Chong, M. S. \& Lim, T. T. 1982 The vortex-shedding process behind two-dimensional bluff bodies. J. Fluid Mech. 116, 77-87.

Smith, R. C. T. 1952 The bending of a semi-infinite strip. Aust. J. Sci. Res. 5, 227-237.

Sturges, L. D. 1986 Stokes flow in a two-dimensional cavity with moving end walls. Phys. Fluids 29, 1731-1734.

Tobak, M. \& Peake, D. J. 1982 Topology of three-dimensional separated flows. Annu. Rev. Fluid Mech. 14, 61-85.

Weiss, R. F. \& Florsheim, B. H. 1965 Flow in a cavity at low reynolds number. Phys. Fluids 8, 1631-1635. 